\title{
Multicellular Interactions in 3D Engineered Myocardial Tissue
}

\author{
Maedeh Zamani ${ }^{1,2}$, Esra Karaca ${ }^{1,2,3}$ and Ngan F. Huang ${ }^{1,2,3 *}$ \\ ${ }^{1}$ School of Medicine, The Stanford Cardiovascular Institute, Stanford University, Stanford, CA, United States, ${ }^{2}$ Department of \\ Cardiothoracic Surgery, Stanford University, Stanford, CA, United States, ${ }^{3}$ Veterans Affairs Palo Alto Health Care System, \\ Palo Alto, CA, United States
}

\section{OPEN ACCESS}

Edited by:

Paolo Madeddu,

University of Bristol, United Kingdom

Reviewed by:

Federico Quaini,

Università degli Studi di Parma, Italy

Claudia Bearzi,

Consiglio Nazionale Delle Ricerche

$(\mathrm{CNR})$, Italy

*Correspondence:

Ngan F. Huang

ngantina@stanford.edu

Specialty section:

This article was submitted to

Cardiovascular Biologics and

Regenerative Medicine,

a section of the journal

Frontiers in Cardiovascular Medicine

Received: 01 May 2018 Accepted: 01 October 2018 Published: 23 October 2018

Citation:

Zamani M, Karaca E and Huang NF (2018) Multicellular Interactions in $3 D$ Engineered Myocardial Tissue. Front. Cardiovasc. Med. 5:147. doi: 10.3389/fcvm.2018.00147
Cardiovascular disease is a leading cause of death in the US and many countries worldwide. Current cell-based clinical trials to restore cardiomyocyte (CM) health by local delivery of cells have shown only moderate benefit in improving cardiac pumping capacity. CMs have highly organized physiological structure and interact dynamically with non-CM populations, including endothelial cells and fibroblasts. Within engineered myocardial tissue, non-CM populations play an important role in $\mathrm{CM}$ survival and function, in part by secreting paracrine factors and cell-cell interactions. In this review, we summarize the progress of engineering myocardial tissue with pre-formed physiological multicellular organization, and present the challenges toward clinical translation.

Keywords: engineered myocardium, cardiovascular tissue engineering, co-culture, cardiomyocyte, endothelial cell, fibroblast, stem cell

\section{INTRODUCTION}

The myocardium is a highly organized complex organ with multicellular structure. Besides the contractile cardiomyocytes (CMs), other important non-myocyte (non-CM) populations include endothelial cells (ECs), fibroblasts (FBs), vascular smooth muscle cells (SMCs), neuronal cells, and immune cells (1). In general, myocardial tissue is composed of $30-40 \%$ CMs and $60-70 \%$ non-CMs, although the cell population ratio varies regionally in the myocardium and across various species, as well as during cardiac development (2-5). Each cellular component has a distinct role in cardiac function. CMs mainly contribute to the conduction of electrical impulses and contraction, while non-CMs are responsible for vascularization, secretion of extracellular matrix (ECM) components and responding to myocardial injuries caused by oxygen depletion during ischemic heart diseases such as myocardial infarction (2). Apart from their individual functions, CMs and non-CMs interact with one another to regulate cellular organization, differentiation, viability and function. By mediating the production and transmission of the electrical, biochemical and mechanical signals in the cardiac microenvironment, non-CMs can regulate the function of CMs (2). The intercommunication among CMs and non-CMs is mediated through cell-cell interactions, soluble signaling proteins secreted by neighboring cells known as paracrine factors, and ECM-mediated crosstalk (6). Understanding the multicellular interactions in myocardial tissue is critically important, not only to provide insight into healthy and diseased cardiac microenvironments, but also to enable the development of cardiomimetic tissue culture systems for cardiovascular regeneration applications. This review presents the recent advances in the development of functional cardiac tissue, with emphasis on the heterocellularity of the engineered constructs, toward resembling the multicellular structure of the myocardial environment (Figure 1). The importance of the cellular components as well as the physicochemical properties of the ECM in recreating native heart-like tissue are explored. 


\section{IN VITRO CO-CULTURE SYSTEMS}

Recent in vitro studies on generation of implantable cardiomimetic units have shown great potential for cardiac regeneration, by developing engineered constructs with cellular, extracellular and biomolecular composition resembling native heart tissue. With respect to the cellular composition, various $\mathrm{CM}$ and non-CM cell types with different origin, age and activation/differentiation state have been employed for developing cardio-mimetic tissue constructs (7). Due to difficulties associated with long-term maintenance of adult CMs in vitro, neonatal CMs, embryonic stem cell-derived $\mathrm{CMs}$ (ESC-CMs) and induced pluripotent stem cell-derived CMs (iPSC-CMs) have been extensively explored in cardiac regeneration $(8,9)$. The use of these less differentiated cell sources signifies the importance of developing a multicellular microenvironment in vitro, in which the interactions with non-CM cells can improve the maturity of CMs in the culture system. Here we describe the progress in recreating the cardiac heterocellular microenvironment using CM-EC and CMFB co-culture systems, and the importance of non-CMs in recapitulating a more biologically relevant microenvironment. We also present an overview of stem cell-based approaches toward developing multicellular tissue constructs, using stem cell-derived CMs/ECs/FBs. Although other cell types like neural cells and immune cells are important in controlling the CM contractility and hypertrophy, these cell types are discussed in greater detail elsewhere $(10,11)$.

\section{Fibroblasts (FBs)}

FBs comprise the major non-CM cellular composition in the adult heart, where each $\mathrm{CM}$ is bordered by at least one $\mathrm{FB}(5,12,13)$. FBs play an essential role in maintaining myocardial structure and mediating $\mathrm{CM}$ function by depositing ECM components $(6,14)$, secreting soluble paracrine factors such as platelet-derived growth factor- $\beta$ (PDGF- $\beta$ ) and basic fibroblast growth factor (bFGF), and propagating electrical signaling through gap junction proteins (e.g., connexin-43), in cell-cell contacts $(15,16)$. FBs can transmit electrical impulses between CMs with over $100 \mu \mathrm{m}$ distance through intercellular gap junctions, contributing in synchronized CM contractions $(15,17,18)$. Under the ischemic conditions, the inflammatory mediators such as transforming growth factor- $\beta$ may induce ECM synthesis by FBs, resulting in cardiac fibrosis (11).

It has been demonstrated that $\mathrm{CM}-\mathrm{FB}$ co-culture can preserve the polarized morphology of $\mathrm{CMs}$ and induce the longterm expression of CM gap junction protein connexin-43 that is responsible for CM-FB heterocellular coupling, resulting in long-term synchronized contraction of engineered cardiac tissue (17). There is evidence that FBs can also modulate the biological properties of $\mathrm{CMs}$ through the secretion of various paracrine factors, without direct cell-cell contact. For example, secretion of periostin by $\mathrm{FBs}$ has been shown to induce the proliferation of $\mathrm{CMs}$ both in vitro and in vivo (19). Soluble vascular cell adhesion molecule-1 (VCAM-1) secreted by cardiac FBs induced proliferation of mouse ESCCMs, resulting in higher number of contractile cells and better propagation of extracellular electrical impulses (20). In addition to cell-cell contact signaling and paracrine factoring, the ECM components (e.g., fibronectin, elastin, and glycoproteins) and matrix metalloproteinases (MMPs) produced by FBs also work interdependently to regulate ECM homeostasis in vivo, with a determining role in cardiac remodeling and scar tissue formation after myocardial infarction $(21,22)$. Although CM-FB co-culture can improve CMs contractility and function, the abundance of FBs needs to be optimized, since increasing the FB population may impair $\mathrm{CM}$ maturation and reduce cardiac conduction speed, action potential propagation and membrane resting potential by the secretion of paracrine factors $(23,24)$.

\section{Endothelial Cells (ECs)}

As a highly metabolic cell type, CMs reside within $2-3 \mu \mathrm{m}$ distance from nearest capillary $(25,26)$. CMs require ECs in the capillary network to provide oxygen and nutrients. ECs have a pivotal role in protecting CMs against ischemic injuries by secreting neuregulin and PDGF- $\beta$ and reducing fibrotic tissue formation after myocardial infarction $(27,28)$. An important goal of CM-EC co-culture is to create a vascularized cardio-mimetic tissue. ECs have also shown to be a dynamic mediator of CM spatial organization, contraction, survival and function in a complex heterocellular physiological platform $(26,29-31)$. ECs in co-culture can promote neovascularization by forming cellular networks within the engineered tissue unit and producing angiogenic cytokines such as vascular endothelial growth factor (VEGF), basic fibroblast growth factor (bFGF), and hepatocyte growth factor (HGF), which can eventually enhance the capillary density of the implanted endothelialized cardiac tissue in vivo (27). ECs can also interact with CMs through autocrine and paracrine signaling factors, such as neuregulin and PDGF- $\beta$ to enhance CM survival (28, 29,31 ), and nitric oxide and endothelin-1 to improve CM contractility $(26,29,32)$. Besides paracrine signaling, ECs direct spatial organization of the CMs through cell-cell contact. CMs formed interconnected organized structures along capillary-like network in the co-culture system, but this organization was not observed when CMs were cultured with EC-conditioned medium (29).

\section{Pluripotent Stem Cell-Derived CMs/FBs/ECs}

Recent advances in efficient differentiation of pluripotent stem cells to cardiac cells represented a new unlimited source of cells for developing cardiac tissues, particularly due to the limited availability of donor primary CMs. However, the survival rate, maturation, and function of these less differentiated cells are highly dependent on the biochemical, mechanical and topographical properties of their microenvironment. The coculture of both embryonic and induced pluripotent stem cellsderived CMs (ESC-CMs and iPSC-CMs) with non-CMs of different sources demonstrated to preserve the CMs phenotype and function through cell-cell contact and biochemical cues (3335). The scaffold-free patches created from human ESC-CMs, ESC-ECs and fibroblasts exhibited electrically-paced contraction and more myocardially-relevant passive mechanical properties, 


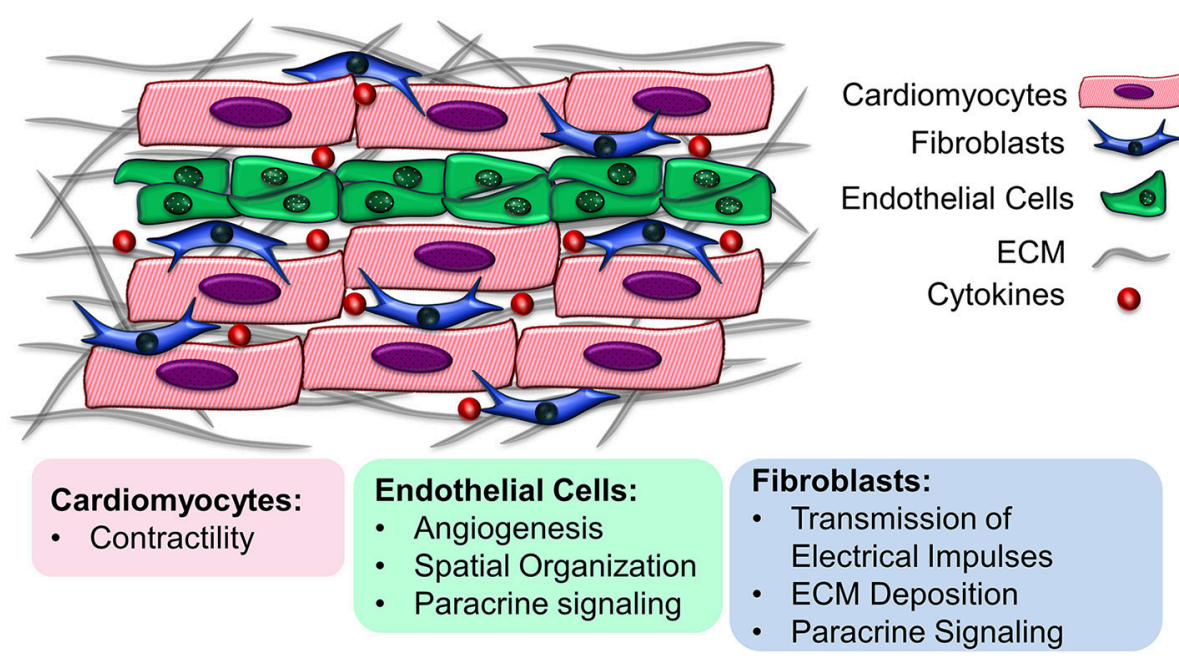

FIGURE 1 | Multicellularity of the myocardial environment. Cardiomyocytes interact with support cells such as endothelial cells and fibroblasts to maintain their cell function. The underlying extracellular matrix also provides instructive cues that regulate cell survival and function.

compared to ESC-CMs in monoculture. Transplantation of multi-culture pre-vascularized tissue constructs showed high engraftment and anastomosis to the animal's coronary artery, unlike the patches consisted of pure ESC-CMs that did not survive after implantation (36). Entirely iPSC-derived cardiac patches composed of iPSC-CMs and iPSC-ECs were developed using aligned nanofibrous scaffolds. The multicellular cardiac patches demonstrated improved maturity of iPSC-CMs, as indicated by increased sarcomeric length and myosin heavy chain gene expression that mimicked mature adult CMs (33). Entirely autologous iPSC-derived multicellular cardiac tissues can potentially address the problems associated with allogeneic cardiac tissues for future clinical applications. Although multicellular interactions are critical for promoting pluripotent stem cell survival and function, the ratio of the non-CMs need to be optimized in the tissue construct. A high fraction of non-CMs may adversely affect iPSC-CMs electrical conduction and maturation through the production of various ECM proteins, i.e., collagen, fibronectin, laminin (33, 34).

\section{ENGINEERING FUNCTIONAL CO-CULTURED TISSUES}

The formation of a functional cardiac construct comprising multi-cellular components requires the cells to be exposed to an in vitro environment that can efficiently support their proliferation, differentiation, organization, and function. Taking advantage of advances in the field of tissue engineering, multi-cellular cardiac constructs have been fabricated over the years using various cell culture techniques and scaffolding materials, to optimize the regenerative ability of the engineered tissue. In general, engineering a biomimetic cardiac tissue in vitro aims at generating a highly aligned, contractile, and vascularized cardiac construct in a three-dimensional (3D) microenvironment. In this section, we present some of the main techniques developed for fabrication of 3D co-cultured cardiac constructs to enhance their cellular organization and function.

\section{Cell Sheet Engineering}

Cell sheet engineering, the technique for constructing 3D functional tissues by layering two-dimensional (2D) cell sheets harvested from thermo-responsive cell culture surfaces, is an efficient approach for developing layered $\mathrm{CM}$ sheets cocultured with non-CMs. A 3D cardiac tissue made through cell sheet technology may resemble dense cellular population and tight interconnection with gap junctions, which facilitates the exchange of biomolecules and ions resulting in electrically synchronized contraction. Synchronized contractions was observed for the layered CMs, indicating the electrical communication between layers of $\mathrm{CMs}$ in $3 \mathrm{D}$ construct (37). However, sufficient oxygen and nutrient diffusion into the layered CMs sheets is critically important, particularly due to the dense cell density in 3D constructs (38). Multicellular 3D platforms were also developed either by layering sheets of heterogeneous cells (27) or mono-cultured layers of different cell types (39). When EC-CM heterogeneous sheets were layered to develop pre-vascularized 3D constructs, it significantly enhanced capillary density in the graft, as compared with mono-cultured cell sheets in vivo (27). Alternating sheets of neonatal CMs and FBs could also form gap junctions between heterogeneous layers, resulting in synchronized beating of the $3 \mathrm{D}$ tissue (39).

\section{Polymer Scaffolds}

Polymer porous scaffolds with a wide range of biochemical, mechanical and topographical properties have been developed for creating cardiac tissues, with the aim of providing a temporary ECM-like support to guide the cells growth, organization 
and function. The native ECM in the heart is a highly anisotropic (parallel-aligned), elastic and fibrillar network, and significant effort has been made to recapitulate this structure $(40,41)$. Polymer scaffolds can mediate the cross-talk between the cells in co-culture through its chemical, mechanical and electrical properties, as well as the architectural design of the scaffold which determines the cellular spatial patterning, cellcell spacing, and the transmission of biomolecular/electrical signals. One advantage of polymer porous scaffolds over cell sheet technology is the ease of engineering 3D constructs. Nanofibrous scaffolds possessing fibrous ECM-like architecture with interconnected pores have been widely explored for generating cardiac tissues $(17,40,42,43)$. The CM-FB and CM-EC co-culture systems on chitosan nanofibers resulted in polarized CMs morphology and maintenance of long-term function (17). Conductive nanofibrous yarns containing carbon nanotubes were incorporated in GelMA hydrogel to produce $3 \mathrm{D}$ composite scaffolds mimicking native cardiac ECM. The coculture of CMs and ECs on the nanofibers yarn and hydrogel construct respectively resulted in aligned CMs morphology in a uniform ECs network, capable of developing pre-vascularized tissue for myocardial regeneration (44). 3D porous scaffolds were also developed through other techniques such as salt leaching (35). Poly(l-lactic acid)/poly(lactide-co-glycolide (PLLA/PLGA) porous sponges seeded with a tri-culture system (i.e., ESCderived $\mathrm{CMs} / \mathrm{FBs} / \mathrm{ECs}$ ) showed higher density of functional vasculature integrated with host myocardial microvasculature, when compared to sponges consisting of mono-cultures of ESCCMs (35).

Since CMs have highly organized physiological structure for driving efficient electromechanical coupling and contractility, much attention has been placed to recreate endogenous CM spatial patterning using instructive polymeric biomaterials. Spatial patterning of CMs on electrospun fibrous scaffolds (45) with aligned organization led to CM alignment along the direction of the fibers, increased contractile strength, and synchronized beating, compared to cells grown on the randomly oriented scaffolds, owing to improved intercellular crosstalk and exchange of ions via gap junctions (46). CM function was further improved when the aligned scaffolds were co-cultured with FBs or ECs. Similar effects of spatial patterning and improved contractile strength has also been reported using iPSC-CMs (33). It was also demonstrated that the alignment of fibrous scaffolds made of elastomeric polyurethane can improve the parallel organization of ESC-CMs and sarcomeric length in a CM-FB co-culture system (42). The roles of cell-cell contact and paracrine factors were further examined when anisotropic scaffolds seeded with rat CMs were co-cultured with scaffolds seeded with fibroblasts with either direct contact or indirect contact (paracrine interaction only). Interestingly, regardless of direct or indirect contact, the presence of FBs improved CM phenotype and aligned sarcomere organization, suggesting that both spatial patterning and paracrine factors are important for CM function (43). Together, these studies suggest that polymer scaffolds and anisotropic spatial patterning promote heterocellular cultures for improved CM function.

\section{Hydrogels}

Hydrogel systems mainly derived from natural sources such as Matrigel (47), fibrin (7), collagen I (48), gelatin methacrylate (GelMA) (49), and peptide hydrogel (29), have been used as substrates to prepare $3 \mathrm{D}$ heterocellular units for cardiac regeneration. To develop hydrogel-based $3 \mathrm{D}$ constructs, a solution of gelling material mixed with cellular components is usually casted into a mold where the gelation process occurs. Although the mechanical properties of hydrogel systems are lower compared to polymer scaffolds, they are normally more compliant than polymers (23). Cellular spreading, the ability to deform the hydrogel, and nutrient exchange are important factors in obtaining a functional contractile tissue construct (50). Studies show that, unlike ESC-CMs cultured in a fibrin-based hydrogel, ESC-CM/FB co-cultures could successfully compact and remodel the hydrogel substrate to generate macroscopically synchronized action potential propagation (23). It was also demonstrated that geometrical properties of the micropatterned hydrogel platform can influence the synchronized contraction of neonatal CMs-FBs co-culture in a GelMa hydrogel $(49,51)$. The authors found that the presence of FBs led to elongation of CMs, increased integrin expression levels, and increased connexin 43 gap junctions, compared to CM-only cultures.

\section{EMERGING TECHNOLOGIES}

In recent years multicellular systems design has been improved with the emerging bioprinting and organ-on-a-chip (OOC) technologies that provide new approaches for basic research and drug discovery. Since the geometry and spatial arrangement of different cell types are important for their function, 3D bioprinting is a useful technology for turning digital designs into precisely organized 3D cultures. Bioprinting uses ECM proteins such as collagen, gelatin, hyaluronic acid, alginate, and decellularized ECMs as bioinks (52) to construct digitally designed $3 \mathrm{D}$ cultures. To overcome the problem of degradation of scaffolds in vivo, bioprinting has also been used to create cardiac tissue consisting of multicellular aggregates and omitting biomaterials altogether (53). Ong et al. created cardiac patches using spheroids of CMs, ECs and FBs that were 3D printed on a needle array and allowed to fuse. They showed that the resulting patch was electrically active and upon implantation in vivo resulted in vascularization and engraftment (54).

Recent research on organoid cultures showed that stem cells have the intrinsic capacity to self-assemble and differentiate into $3 \mathrm{D}$ tissues that can replicate some of the properties of the native organ, which suggests that it may not be necessary to $3 \mathrm{D}$ print the entire tissue. The cardiac organoids developed so far remain in the more immature stages and resemble fetal heart (55). Bioprinting can be employed to guide the cardiac organoid formation by creating patterns and guidance cues in its microenvironment (56).

Some of the limitations of traditional static 3D cultures such as lack of geometric organization, absence of a clear tissue interface, and the accumulation of secreted compounds into spent media can be overcome in a microfluidic platform. 
Microfluidic platforms enable tissues of different cell types to be separated from one another by porous membranes to allow for molecular crosstalk. These OOC platforms allow for longer term cultures, owing to the circulating factors, nutrients and oxygen in the microfluidic system. The circulation of media in the capillaries give rise to mechanical factors such as fluid shear stress and allows for the monitoring of secreted factors and metabolites in real-time. The microfluidic chips also make it possible to investigate changes in the morphology and migration of cells (57). This approach has been used to create myocardial OOCs using CMs and ECs derived from human iPSCs to create models for drug screening and disease modeling $(58,59)$.

\section{LIMITATIONS AND FUTURE DIRECTIONS}

In vitro co-culture systems play a significant role in advances in cardiac tissue engineering over the last years. Using heterocellular engineered cardiac tissues, we are closer to developing functional vascularized tissues by taking advantage of complex and dynamic crosstalk between various types of cells and their interactions with ECM. However, extensive studies are yet required to fully understand the distinct roles of each individual cellular component and their interactions, to optimize engineered vascularized cardiac tissue. The in vitro co-culture systems are capable of creating physiologically relevant cardiac tissues for regenerative purposes. However, the read-out of multicellular systems is highly complexed and more innovative cell culture systems and characterization techniques are still required to precisely understand the heterocellular communication at a single-cell level to progress in the field. Although 3D scaffolds may better recapitulate the native heart ECM network, the difficulties associated with oxygen and nutrient diffusion, cell

\section{REFERENCES}

1. Wanjare M, Huang NF. Regulation of the microenvironment for cardiac tissue engineering. Regen Med. (2017) 12:187-201. doi: 10.2217/rme-2016-0132

2. Kofron CM, Mende U. In vitro models of the cardiac microenvironment to study myocyte and non-myocyte crosstalk: Bioinspired approaches beyond the polystyrene dish. J Physiol. (2017) 595:3891-905. doi: 10.1113/JP273100

3. Banerjee I, Fuseler JW, Price RL, Borg TK, A. BT. Determination of cell types and numbers during cardiac development in the neonatal and adult rat and mouse. Am J Physiol Heart Circ Physiol. (2007) 293:H1883-91. doi: 10.1152/ajpheart.00514.2007.-Cardiac

4. Zak R. Development and proliferative capacity of cardiac muscle cells. Circ Res. (1974) 35(Suppl. 2):17-26.

5. Nag A. Study of non-muscle cells of the adult mammalian heart: A fine structural analysis and distribution. Cytobios (1979) 28:41-61.

6. Zhang P, Su J, Mende U. Cross talk between cardiac myocytes and fibroblasts: From multiscale investigative approaches to mechanisms and functional consequences. Am J Physiol Heart Circ Physiol. (2012) 303:H1385-96. doi: 10.1152/ajpheart.01167.2011.-The

7. Liau B, Jackman CP, Li Y, Bursac N. Developmental stage-dependent effects of cardiac fibroblasts on function of stem cell-derived engineered cardiac tissues. Sci Rep. (2017) 7:42290. doi: 10.1038/srep42290

8. Karakikes I, Ameen M, Termglinchan V, Wu JC. Human induced pluripotent stem cell-derived cardiomyocytes: Insights into molecular, cellular, and functional phenotypes. Circ Res. (2015) 117:80-8. doi: 10.1161/CIRCRESAHA.117.305365 infiltration and viability, vascularization, and degree of scaffold remodeling may impair the clinical translation of multicellular constructs made by using 3D scaffolds. The source of the cells as well as the age, differentiation state and cell ratios of the co-culture platforms are important considerations influencing the functions of engineered vascularized cardiac tissues. Despite advances in employing ESC-CMs and iPSC-CMs for cardiac regeneration, it is critical to design cellular microenvironment to maintain characteristics of these differentiated cells by introducing relevant electrical, molecular, mechanical and topographical cues in long-term culture systems. The use of advanced materials such as conductive scaffolds may also facilitate the electrophysiological communication among the cells of multicellular environment. As more advanced techniques emerge in the field of tissue engineering, more biologically relevant native myocardial-like tissues will be developed for regenerative applications.

\section{AUTHOR CONTRIBUTIONS}

$\mathrm{MZ}$ and $\mathrm{NH}$ conceived the content of the manuscript. MZ and EK analyzed the literature and wrote the manuscript. $\mathrm{NH}$ critically reviewed and revised the manuscript. All authors read and approved the submitted version.

\section{ACKNOWLEDGMENTS}

This work was supported in part by grants to $\mathrm{NH}$ from the US National Institutes of Health (R01 HL127113 and R01HL142718), the Department of Veterans Affairs (1I01BX002310), and the California Institute for Regenerative Medicine (10603).

9. Feinberg AW, Ripplinger CM, van der Meer P, Sheehy SP, Domian I, Chien $\mathrm{KR}$, et al. Functional differences in engineered myocardium from embryonic stem cell-derived versus neonatal cardiomyocytes. Stem Cell Rep. (2013) 1:387-96. doi: 10.1016/j.stemcr.2013.10.004

10. Franzoso M, Zaglia T, Mongillo M. Putting together the clues of the everlasting neuro-cardiac liaison. Biochim Biophys Acta (2016) 1863(7 Pt B):1904-15. doi: 10.1016/j.bbamcr.2016.01.009

11. Kamo T, Akazawa H, Komuro I. Cardiac nonmyocytes in the hub of cardiac hypertrophy. Circ Res. (2015) 117:89-98. doi: 10.1161/CIRCRESAHA.117.305349

12. Camelliti P, Borg TK, Kohl P. Structural and functional characterisation of cardiac fibroblasts. Cardiovasc Res. (2005) 65:40-51. doi: 10.1016/j.cardiores.2004.08.020

13. Kohl P, Camelliti P, Burton FL, Smith GL. Electrical coupling of fibroblasts and myocytes: Relevance for cardiac propagation. J Electrocardiol. (2005) 38:45-50. doi: 10.1016/j.jelectrocard.2005.06.096

14. Ogle BM, Bursac N, Domian I, Huang NF, Menasche P, Murry CE, et al. Distilling complexity to advance cardiac tissue engineering. Sci Transl Med. (2016) 8:342ps313. doi: 10.1126/scitranslmed.aad2304

15. Gaudesius G, Miragoli M, Thomas S, Rohr S. Coupling of cardiac electrical activity over extended distances by fibroblasts of cardiac origin. Circ Res. (2003) 93:421-8. doi: 10.1161/01.RES.0000089258.40661.0C

16. Kizana E, Ginn SL, Smyth CM, Boyd A, Thomas SP, Allen DG, et al. Fibroblasts modulate cardiomyocyte excitability: implications for cardiac gene therapy. Gene Ther. (2006) 13:1611-5. doi: 10.1038/sj.gt. 3302813 
17. Hussain A, Collins G, Yip D, Cho CH. Functional 3-d cardiac co-culture model using bioactive chitosan nanofiber scaffolds. Biotechnol Bioeng. (2013) 110:637-47. doi: 10.1002/bit.24727/abstract

18. Kohl P, Gourdie RG. Fibroblast-myocyte electrotonic coupling: does it occur in native cardiac tissue? J Mol Cell Cardiol. (2014) 70:37-46. doi: 10.1016/j.yjmcc.2013.12.024

19. Kühn B, del Monte F, Hajjar RJ, Chang YS, Lebeche D, Arab S, et al. Periostin induces proliferation of differentiated cardiomyocytes and promotes cardiac repair. Nat Med. (2007) 13:962-9. doi: 10.1038/ nm1619

20. Iwamiya $\mathrm{T}$, Matsuura K, Masuda S, Shimizu T, Okano T. Cardiac fibroblast-derived vcam-1 enhances cardiomyocyte proliferation for fabrication of bioengineered cardiac tissue. Regener Ther. (2016) 4:92-102. doi: 10.1016/j.reth.2016.01.005

21. Souders CA, Bowers SLK, Baudino TA. Cardiac fibroblast: the renaissance cell. Circ Res. (2009) 105:1164-76. doi: 10.1161/CIRCRESAHA.109.209809

22. Carver W, Nagpal ML, Nachtigal M, Borg TK, Terracio L. Collagen expression in mechanically stimulated cardiac fibroblasts. Circ Res. (1991) 69:116-22.

23. Liau B, Christoforou N, Leong KW, Bursac N. Pluripotent stem cell-derived cardiac tissue patch with advanced structure and function. Biomaterials (2011) 32:9180-7. doi: 10.1016/j.biomaterials.2011.08.050

24. Pedrotty DM, Klinger RY, Kirkton RD, Bursac N. Cardiac fibroblast paracrine factors alter impulse conduction and ion channel expression of neonatal rat cardiomyocytes. Cardiovasc Res. (2009) 83:688-97. doi: 10.1093/cvr/ cvp164

25. Shah AM. Paracrinemodulation of heart cell function by endothelial cells. Cardiovasc Res. (1996) 31:847-67.

26. Hsieh PCH, Davis ME, Lisowski LK, Lee RT. Endothelialcardiomyocyte interactions in cardiac development and repair. Annu Rev Physiol. (2006) 68:51-66. doi: 10.1146/annurev.physiol.68.040104.124629

27. Sekine H, Shimizu T, Hobo K, Sekiya S, Yang J, Yamato M, et al. Endothelial cell coculture within tissue-engineered cardiomyocyte sheets enhances neovascularization and improves cardiac function of ischemic hearts. Circulation (2008) 118(14 Suppl):S145-152. doi: 10.1161/CIRCULATIONAHA.107.757286

28. Edelberg JM, Aird WC, Wu W, Rayburn H, Mamuya WS, al. e. Pdgf mediates cardiac microvascular communication. J Clin Invest. (1998) 102:837-43.

29. Narmoneva DA, Vukmirovic R, Davis ME, Kamm RD, Lee RT. Endothelial cells promote cardiacmyocyte survival and spatial reorganization: implications for cardiac regeneration. Circulation (2004) 110:962-8. doi: 10.1161/01.CIR.0000140667.37070.07

30. Burridge PW, Metzler SA, Nakayama KH, Abilez OJ, Simmons CS, Bruce MA, et al. Multi-cellular interactions sustain long-term contractility of human pluripotent stem cell-derived cardiomyocytes. Am J Transl Res. (2014) 6:724-35.

31. Kuramochi Y, Cote GM, Guo X, Lebrasseur NK, Cui L, Liao R, et al. Cardiac endothelial cells regulate reactive oxygen speciesinduced cardiomyocyte apoptosis through neuregulin-1beta/erbb4 signaling. J Biol Chem. (2004) 279:51141-7. doi: 10.1074/jbc.M4086 62200

32. Lim SL, Lam CS, Segers VF, Brutsaert DL, De Keulenaer GW. Cardiac endothelium-myocyte interaction: clinical opportunities for new heart failure therapies regardless of ejection fraction. Eur Heart J. (2015) 36:2050-60. doi: 10.1093/eurheartj/ehv132

33. Wanjare M, Hou LQ, Nakayama KH, Kim JJ, Mezak NP, Abilez OJ, et al. Anisotropic microfibrous scaffolds enhance the organization and function of cardiomyocytes derived from induced pluripotent stem cells. Biomater Sci. (2017) 5:1567-78. doi: 10.1039/c7bm00323d

34. Iseoka H, Miyagawa S, Fukushima S, Saito A, Masuda S, Yajima $\mathrm{S}$, et al. Pivotal role of non-cardiomyocytes in electromechanical and therapeutic potential of induced pluripotent stem cell-derived engineered cardiac tissue. Tissue Eng Part A (2018) 24:287-300. doi: 10.1089/ten.tea. 2016.0535

35. Lesman A, Habib M, Caspi O, Gepstein A, Arbel G, Levenberg S, et al. Transplantation of a tissue-engineered human vascularized cardiac muscle. Tissue Eng Part A (2010) 16:115-25. doi: 10.1089/ten.TEA.2009.0130

36. Stevens KR, Kreutziger KL, Dupras SK, Korte FS, Regnier M, Muskheli V, et al. Physiological function and transplantation of scaffold-free and vascularized human cardiac muscle tissue. Proc Natl Acad Sci USA. (2009) 106:16568-73. doi: $10.1073 /$ pnas.0908381106

37. Reinlib L, Field L. Cell transplantation as future therapy for cardiovascular disease. A workshop of the national heart lung and blood institute. Circulation (2000) 101:E182-187.

38. Shimizu T, Yamato M, Kikuchi A, Okano T. Cell sheet engineering for myocardial tissue reconstruction. Biomaterials (2003) 24:2309-16. doi: 10.1016/s0142-9612(03)00110-8

39. Haraguchi Y, Shimizu T, Yamato M, Okano T. Electrical interaction between cardiomyocyte sheets separated by non-cardiomyocyte sheets in heterogeneous tissues. J Tissue Eng Regen Med. (2010) 4:291-9. doi: $10.1002 /$ term.241

40. Capulli AK, MacQueen LA, Sheehy SP, Parker KK. Fibrous scaffolds for building hearts and heart parts. Adv Drug Deliv Rev. (2016) 96:83-102. doi: 10.1016/j.addr.2015.11.020

41. Neal RA, Jean A, Park H, Wu PB, Hsiao J, Engelmayr GC Jr, et al. Three-dimensional elastomeric scaffolds designed with cardiac-mimetic structural and mechanical features. Tissue Eng Part A (2013) 19:793-807. doi: 10.1089/ten.tea.2012.0330

42. Parrag IC, Zandstra PW, Woodhouse KA. Fiber alignment and coculture with fibroblasts improves the differentiated phenotype of murine embryonic stem cell-derived cardiomyocytes for cardiac tissue engineering. Biotechnol Bioeng. (2012) 109:813-22. doi: 10.1002/bit. 23353

43. Suhaeri M, Subbiah R, Kim SH, Kim CH, Oh SJ, Kim SH, et al. Novel platform of cardiomyocyte culture and coculture via fibroblast-derived matrix-coupled aligned electrospun nanofiber. ACS Appl Mater Interf. (2017) 9:224-35. doi: 10.1021/acsami.6b14020

44. Wu YB, Wang L, Guo BL, Ma PX. Interwoven aligned conductive nanofiber yarn/hydrogel composite scaffolds for engineered 3d cardiac anisotropy. Acs Nano (2017) 11:5646-59. doi: 10.1021/acsnano.7b01062

45. Kim J, Staunton JR, Tanner K. Independent control of topography for $3 d$ patterning of the ecm microenvironment. Adv Mater (2016) 28:132-7. doi: 10.1002/adma.201503950

46. Hsiao CW, Bai MY, Chang Y, Chung MF, Lee TY, Wu CT, et al. Electrical coupling of isolated cardiomyocyte clusters grown on aligned conductive nanofibrous meshes for their synchronized beating. Biomaterials (2013) 34:1063-72. doi: 10.1016/j.biomaterials.2012.10.065

47. Li Y, Asfour H, Bursac N. Age-dependent functional crosstalk between cardiac fibroblasts and cardiomyocytes in a $3 \mathrm{~d}$ engineered cardiac tissue. Acta Biomater. (2017) 55:120-30. doi: 10.1016/j.actbio.2017.04.027

48. Ou DB, He Y, Chen R, Teng JW, Wang HT, Zeng D, et al. Three-dimensional co-culture facilitates the differentiation of embryonic stem cells into mature cardiomyocytes. J Cell Biochem. (2011) 112:3555-62. doi: 10.1002/jcb. 23283

49. Saini H, Navaei A, Van Putten A, Nikkhah M. 3d cardiac microtissues encapsulated with the co-culture of cardiomyocytes and cardiac fibroblasts. Adv Healthc Mater (2015) 4:1961-71. doi: 10.1002/adhm.2015 00331

50. Hirt MN, Hansen A, Eschenhagen T. Cardiac tissue engineering: State of the art. Circ Res. (2014) 114:354-67. doi: 10.1161/CIRCRESAHA.114. 300522

51. Camci-Unal G, Zorlutuna P, Khademhosseini A.(2013) Fabrication of microscale hydrogels for tissue engineering applications. In: Forgacs G, Sun W, editors. Biofabrication: Micro- and Nano-Fabrication, Printing, Patterning and Assemblies (Elsevier). 59-80. doi: 10.1016/b978-1-4557-2852-7.00004-4

52. Jang J, Park HJ, Kim SW, Kim H, Park JY, Na SJ, et al. 3d printed complex tissue construct using stem cell-laden decellularized extracellular matrix bioinks for cardiac repair. Biomaterials (2017) 112264-274. doi: 10.1016/j.biomaterials.2016.10.026

53. Rogozhnikov D, O’Brien PJ, Elahipanah S, Yousaf MN. Scaffold free bio-orthogonal assembly of 3-dimensional cardiac tissue via cell surface engineering. Sci Rep. (2016) 6:39806. doi: 10.1038/srep 39806

54. Ong CS, Fukunishi T, Zhang HT, Huang CY, Nashed A, Blazeski A, et al. Biomaterial-free three-dimensional bioprinting of cardiac tissue using human induced pluripotent stem cell derived cardiomyocytes. Sci Rep. (2017) 7:4566. doi: 10.1038/s41598-017-05018-4 
55. Voges HK, Mills RJ, Elliott DA, Parton RG, Porrello ER, Hudson JE. Development of a human cardiac organoid injury model reveals innate regenerative potential. Development (2017) 144:1118-27. doi: $10.1242 /$ dev. 143966

56. Hoang P, Wang J, Conklin BR, Healy KE, Ma Z. Generation of spatial-patterned early-developing cardiac organoids using human pluripotent stem cells. Nat Protocols (2018) 13:723-37. doi: 10.1038/nprot. 2018.006

57. Bhatia SN, Ingber DE. Microfluidic organs-on-chips. Nat Biotechnol. (2014) 32:760-72. doi: 10.1038/nbt.2989

58. Ellis BW, Acun A, Can UI, Zorlutuna P. Human ipsc-derived myocardiumon-chip with capillary-like flow for personalized medicine. Biomicrofluidics (2017) 11:024105. doi: 10.1063/1.4978468

59. Zhang YS, Arneri A, Bersini S, Shin SR, Zhu K, GoliMalekabadi Z, et al. Bioprinting 3d microfibrous scaffoldsfor $\begin{array}{ll}\text { engineering } & \text { endothelialized myocardium and heart-on-a-chip. } \\ \text { Biomaterials } & \text { (2016) 110:45-59. doi: 10.1016/j.biomaterials.2016. }\end{array}$ 09.003

Conflict of Interest Statement: The authors declare that the research was conducted in the absence of any commercial or financial relationships that could be construed as a potential conflict of interest.

Copyright (c) 2018 Zamani, Karaca and Huang. This is an open-access article distributed under the terms of the Creative Commons Attribution License (CC BY). The use, distribution or reproduction in other forums is permitted, provided the original author(s) and the copyright owner(s) are credited and that the original publication in this journal is cited, in accordance with accepted academic practice. No use, distribution or reproduction is permitted which does not comply with these terms. 